\title{
Nanoscale flow cytometry of patient plasma for the detection of prostate cancer-associated extracellular vesicles
}

AUTHORS: Andrew C. Poon ${ }^{1,2}$, Johanna Garzon ${ }^{3}$, Sabine Brett², Matthew Lowerison ${ }^{4}$, Karla Williams ${ }^{3}$,

Hon S. Leong ${ }^{1.2 .3}$

\section{INSTITUTION:}

1. Department of Pathology and Laboratory Medicine, University of Western Ontario, London, ON

2. Department of Microbiology and Immunology, University of Western Ontario, London, ON

3. Department of Surgery, Division of Urology, Lawson Health Research Institute, London, ON

4. Department of Medical Biophysics, University of Western Ontario, London, ON

EDITOR'S NOTE: The following is a project nominated by representatives from the Western Student Research Conference (WSRC) for publication within the STEM Fellowship Journal. The WSRC is an annual multidisciplinary undergraduate research conference which is open to students across Ontario.

\section{Nanoscale Flow Cytometry (A50+) as a Novel Fluid Biopsy for Early Prostate Cancer Detection}

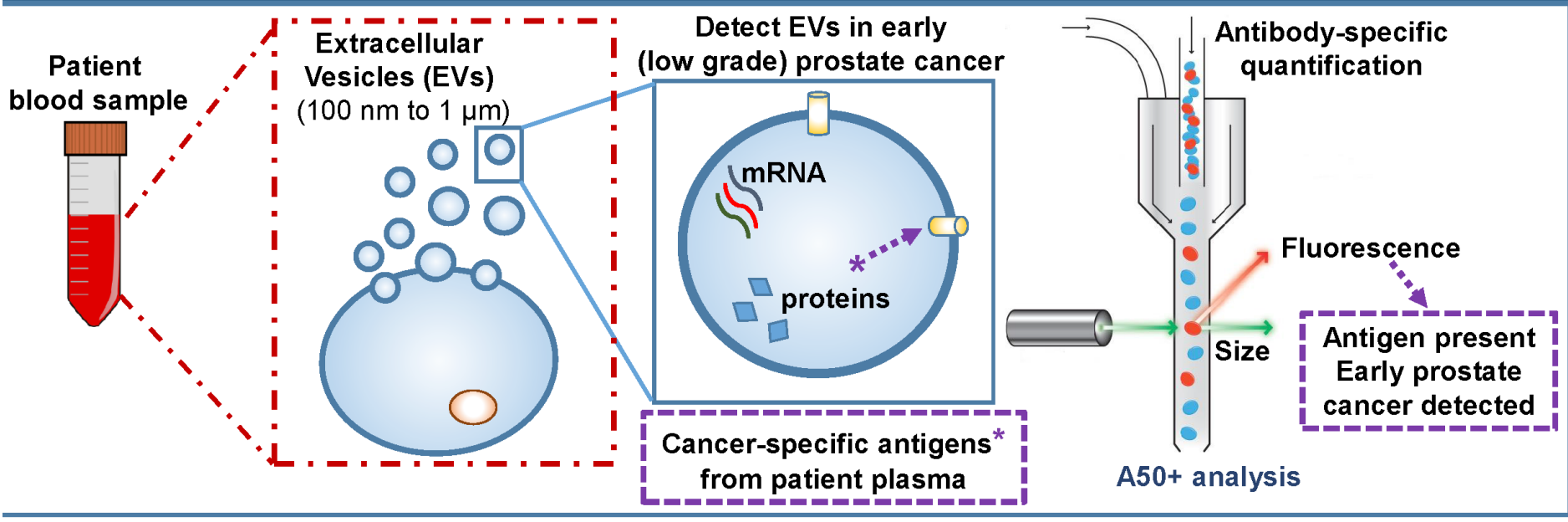

\section{Abstract}

Introduction:

Prostate cancer is the predominant cancer in men, affecting one in seven men during their lifetime. Current tests for prostate cancer include the digital rectal exam and the prostate-specific antigen (PSA) test. Extracellular vesicles (EVs) are submicron particles that participate in intercellular cross-talk by releasing cell mediators such as microRNA, carbohydrates and proteins. While they are known to express the broad tetraspanin family of proteins, i.e. CD9/CD63, prostate cancer-derived EVs have also been found to express PSA and six transmembrane epithelial antigen of the prostate (STEAP1). Traditionally, scientists have purified these EVs through ultracentrifugation. Here we propose a tandem purification of patient plasma, followed by nanoscale flow cytometry (A50+) as a novel method to detect tumour-derived EVs.

\section{Materials and Methods:}

Plasma was obtained from healthy volunteers, patients with benign prostatic hyperplasia (BPH), and patients with metastatic prostate cancer. CD9, CD63, PSA and STEAP1 were used as primary antibodies for the purification of EVs from neat plasma. To perform the puri- 
fication in tandem, Protein G immunoprecipitation using CD9 and PSA was carried out first, followed by immunoaffinity purification with biotinylated CD63 and STEAP1. First and second elutions were collected for the enumeration of dual positive events by A50+. Initial histogram overlays and bivariate plots of neat and purified plasma were computed, then exported as comma-separated values for mathematical modelling by MATLAB.

\section{Results:}

Strong dual positive EV populations from patient plasma were optimised, demonstrating that the method enriches tumour-derived EVs from neat samples of patient plasma. This was observed for HVs, patients with benign prostatic hyperplasia, and prostate cancer patients with Gleason 4+4. Enrichment in these purified samples was measured by A50+ and demonstrated by overlaying purified and non-purified histoplots by MATLAB. Additional results show that PSA and STEAP1 can be adapted to detect single or dual positive populations of tumour-associated EVs in prostate cancer patients.

\section{Discussions and Conclusions:}

This study suggests that tandem purification of tumour-associated EVs and A50+ analysis from plasma of prostate cancer patients can lead to earlier diagnosis and risk stratification, compared to traditional screening tests and aspiration cytology. Future studies will be directed toward optimizing this detection method for markers of other cancer types to achieve better outcomes in cancer detection and prognosis.

\section{Keywords}

Prostate cancer; extracellular vesicles; fluid biopsy; nanoscale flow cytometry; prostate-specific antigen; tetraspanins 